\title{
A Scoring System to Predict Arm Lymphedema Risk for Individual Chinese Breast Cancer Patients
}

\author{
Ling Wang ${ }^{a, b}$ Hui-Ping Li ${ }^{b}$ An-Nuo Liub De-Bin Wang ${ }^{c}$ Ya-Juan Yang ${ }^{b}$ Yan-Oin Duan ${ }^{d}$ \\ Qing-Na Zhange
}

a Department of Nursing, Wannan Medical College, Wuhu, China;

bSchool of Nursing, Anhui Medical University, Heifei, China;

'School of Health Services Management, Anhui Medical University, Heifei, China;

dPeople's Hospital of Huaibei City, Huaibei, China;

${ }^{\mathrm{e}}$ The First Affiliated Hospital of Anhui Medical University, Heifei, China

\section{Keywords \\ Breast cancer · Lymphedema scoring system}

\section{Summary}

Background: Lymphedema (LE) is recognized as a common complication after axillary lymph node dissection (ALND). Numerous studies have attempted to identify risk factors for LE. However, it is difficult to predict the probability of LE for an individual patient. The purpose of this study was to construct a scoring system for predicting the probability of LE after ALND for Chinese breast cancer patients. Patients and Methods: 358 breast cancer patients were surveyed and followed for 12 months. LE was defined by circumferential measurement. Univariate and multivariate logistic regression analyses were used to screen risk factors of LE. Based on this, ß-coefficient of each risk factor was translated into a prognostic score and the scoring system was constructed. The area under the receiver operating characteristic curve (AUC) and calibration were calculated as an index for the predictive value of the scoring system. The model was internally validated using bootstrapping techniques. Results: The incidence rate of LE was $31.84 \%$. Variables associated with LE and their corresponding score in the scoring system were: the level of ALND (level I =0, level II = 1, level III = 2), history of hypertension (yes $=1$, no $=0$ ), surgery on dominant arm (yes $=1$, no $=0$ ), radiotherapy (yes $=2$, no $=0$ ), and surgical infec-

Ling Wang and An-nuo Liu contributed equally to this work and should be considered co-first authors. tion/seroma/early edema (yes $=2$, no $=0$ ). The probability of LE was predicted according to the total risk scores. The system had good discrimination, with an AUC at 0.877 . If a cut-off value of 3 was used, the sensitivity was $81.20 \%$ and the specificity was $80.90 \%$. An individual whose total risk score was higher than 3 was recognized as being at risk for LE. On internal validation, the bootstrap-corrected predictive accuracy was 0.798 . The model demonstrated excellent calibration in the development set and internal validation. Conclusions: Our scoring system could be a simple and easy tool for physicians to estimate the risk of LE.

(c) 2016 S. Karger GmbH, Freiburg

\section{Introduction}

Breast cancer is the most common cancer among women worldwide with approximately 1.3 million people developing breast cancer each year $[1,2]$. At present, routine surgical treatments of breast cancer are either lumpectomy or mastectomy combined with sentinel lymph node (SLN) surgery. Although the results of the ACOSOG Z0011 Trial [3] suggest that women with clinically node negative T1-T2 tumors or fewer than 3 involved SLN cannot benefit from axillary lymph node dissection (ALND), for patients with positive SLN, ALND remains the standard practice. Complete ALND is associated with a nearly 3-fold increased risk of lymphedema (LE) $[4,5]$. As breast cancer survival rate has increased dramatically, with $89 \%$ of these women surviving 5 years [2], LE will potentially impact more women. 
Table 1. Characteristics and univariate analysis of risk factors among cases and controls

\begin{tabular}{|c|c|c|c|}
\hline & Case & Control & $\mathrm{p}$ value \\
\hline Patients, $\mathrm{n}$ & 114 & 244 & \\
\hline \multicolumn{4}{|l|}{ Age, n (\%) } \\
\hline$<60$ years & $98(85.96)$ & $217(88.93)$ & \multirow[t]{2}{*}{0.456} \\
\hline$\geq 60$ years & $16(14.04)$ & $27(11.07)$ & \\
\hline \multicolumn{4}{|l|}{ Menopausal, n (\%) } \\
\hline Yes & $36(31.58)$ & $83(34.02)$ & \multirow[t]{2}{*}{0.692} \\
\hline No & $78(68.42)$ & $161(65.98)$ & \\
\hline \multicolumn{4}{|l|}{ BMI $\left(\mathrm{kg} / \mathrm{m}^{2}\right), \mathrm{n}(\%)$} \\
\hline$<25$ & $86(75.44)$ & $182(74.59)$ & \multirow[t]{2}{*}{0.915} \\
\hline$\geq 25$ & $28(24.56)$ & $62(25.41)$ & \\
\hline \multicolumn{4}{|l|}{ Elevated BMI $\left(\mathrm{kg} / \mathrm{m}^{2}\right), \mathrm{n}(\%)$} \\
\hline$<1$ & $68(59.65)$ & $164(67.21)$ & \multirow[t]{3}{*}{0.159} \\
\hline $1-2$ & $20(17.54)$ & $41(16.80)$ & \\
\hline$>2$ & $26(22.81)$ & 39 (15.99) & \\
\hline \multicolumn{4}{|l|}{ History of hypertension, n (\%) } \\
\hline Yes & $26(22.35)$ & $20(8.05)$ & \multirow[t]{2}{*}{0.000} \\
\hline No & $88(77.65)$ & $224(91.95)$ & \\
\hline \multicolumn{4}{|l|}{ Staging (UICC), n (\%) } \\
\hline 0 & $2(1.75)$ & $4(1.64)$ & \multirow[t]{4}{*}{0.172} \\
\hline I & $9(7.89)$ & $30(12.30)$ & \\
\hline II & $76(66.67)$ & $178(72.95)$ & \\
\hline III & $27(23.69)$ & $32(13.11)$ & \\
\hline \multicolumn{4}{|l|}{ ALN status , n (\%) } \\
\hline Negative & $67(58.77)$ & $201(82.38)$ & \multirow[t]{3}{*}{0.000} \\
\hline Positive (1-4) & $31(27.19)$ & $34(13.93)$ & \\
\hline Positive (> 4) & $16(14.04)$ & $9(3.69)$ & \\
\hline \multicolumn{4}{|l|}{ Type of surgery, n (\%) } \\
\hline Mastectomy & $107(93.86)$ & $230(94.26)$ & \multirow[t]{3}{*}{0.965} \\
\hline Conservative & $4(3.51)$ & $7(2.87)$ & \\
\hline Mastectomy with immediate BR & $3(2.63)$ & $7(2.87)$ & \\
\hline \multicolumn{4}{|l|}{ Surgery on dominant arm, n (\%) } \\
\hline Yes & $70(61.40)$ & $68(27.87)$ & \multirow[t]{2}{*}{0.000} \\
\hline No & $44(38.60)$ & $176(72.13)$ & \\
\hline \multicolumn{4}{|c|}{$\begin{array}{l}\text { Surgical infection/ early edema/ seroma, } \\
\mathrm{n}(\%)\end{array}$} \\
\hline Yes & $48(42.11)$ & $28(11.48)$ & \multirow[t]{2}{*}{0.000} \\
\hline No & $66(57.89)$ & $216(88.52)$ & \\
\hline Level of ALND, n (\%) & & & \\
\hline I & $1(0.88)$ & $6(2.46)$ & 0.000 \\
\hline II & $39(34.21)$ & $148(60.66)$ & \\
\hline III & $74(64.91)$ & $90(36.88)$ & \\
\hline No. of lymph nodes dissected, $\mathrm{n}(\%)$ & & & \\
\hline$<10$ & $16(14.03)$ & $69(28.28)$ & 0.001 \\
\hline $10-20$ & $76(66.67)$ & $157(64.34)$ & \\
\hline$>20$ & $22(19.30)$ & $18(7.38)$ & \\
\hline Chemotherapy, n (\%) & & & \\
\hline Yes & $110(96.49)$ & $232(95.08)$ & 0.771 \\
\hline No & $4(3.51)$ & $12(4.92)$ & \\
\hline Radiotherapy, n (\%) & & & \\
\hline $\begin{array}{l}\text { Yes (breast/chest wall or lymph } \\
\text { node basins) }\end{array}$ & $47(41.23)$ & $32(13.11)$ & 0.000 \\
\hline No & $67(58.77)$ & $212(86.89)$ & \\
\hline Hormonal therapy, n (\%) & & & \\
\hline Yes & $40(35.09)$ & $74(30.33)$ & 0.386 \\
\hline No & $74(64.91)$ & $170(69.67)$ & \\
\hline
\end{tabular}

$\mathrm{BMI}=$ body mass index, $\mathrm{ALN}(\mathrm{D})$ = axillary lymph node (dissection), BR = breast reconstruction 
Table 2. Multivariate logistic regression analysis testing for independent effects

\begin{tabular}{lllclll}
\hline Predictor variable & B & S.E. & Wald & p value & OR & $95 \%$ CI \\
\hline History of hypertension & 1.103 & 0.366 & 7.648 & 0.006 & 2.753 & $1.343-5.642$ \\
Surgery on dominant arm & 0.898 & 0.452 & 3.946 & 0.047 & 2.454 & $1.012-5.948$ \\
Level of ALND & & & & & & \\
$\quad$ I & 0 & 0 & 10.760 & 0.005 & 1 & \\
$\quad$ II & 0.998 & 0.398 & 6.272 & 0.012 & 1.712 & $1.242-5.922$ \\
$\quad$ III & 1.772 & 0.665 & 7.103 & 0.008 & 5.844 & $1.59-21.660$ \\
Radiotherapy to lymph node basin or & 1.445 & 0.369 & 15.536 & 0.000 & 4.285 & $2.078-8.835$ \\
breast/chest wall & & & & & & \\
Surgical infection /seroma /early edema & 1.511 & 0.379 & 15.920 & 0.000 & 4.532 & $2.157-9.520$ \\
\hline
\end{tabular}

ALND = axillary lymph node dissection, $\beta=\beta$-coefficient, S.E. $=$ standard error, $\mathrm{OR}=$ odds ratio, $\mathrm{CI}=$ confidence interval.
LE is characterized by an abnormal accumulation of lymph in the interstitial spaces, leading to persistent swelling in the affected arm, shoulder, neck, breast, or thoracic region, or any combination of these [6]. LE has become a high-impact problem that profoundly impairs the quality of life in breast cancer survivors [7]. The reported prevalence of LE varies with the length of follow-up, the standardized definitions, measurement techniques, or other factors. Estimates for the incidence of LE in previous studies vary from $6.7 \%$ to $62.5 \%[8,9]$. The onset of LE may occur at any time after breast cancer surgery [10], and there is no safe period after which an individual is no longer at risk for developing LE [6].

LE is a chronic, potentially disfiguring condition requiring early detection and management [11]. Therefore, identifying factors that place a breast cancer survivor at higher risk for developing LE is paramount. Studies have associated various risk factors with LE, such as the level of ALND, age, and radiotherapy [12]. While each of these factors contributes significantly and independently to LE, the combined effects of these factors on individual outcome have seldom been addressed. To aid these studies, nnew decisional tools have been proposed based on statistical approaches, e.g. nomograms and predicting tables. Prediction tables have the benefit of providing outcome measures in an easily interpretable format that can be presented and applied to any patient in the clinic. The aim of our study was to create a scoring system for predicting the individual probability of LE after ALND for Chinese breast cancer patients.

\section{Patients and Methods}

Patients who had been diagnosed with breast cancer and underwent ALND (level I, II, or III) between January 2009 and January 2011 at the First Affiliated Hospital of Anhui Medical University were eligible for this study. Other inclusion criteria included being over 18 years of age, being able and willing to give consent, and having no history of other cancers. Patients with systemic disease, previous contralateral or a synchronous bilateral breast cancer, previous surgery or injury performed in the axilla or other conditions that could cause edema (e.g. congestive heart failure, or renal disease) were excluded. All Patients were followed in the clinic for 12 months, and attempts were made to contact any women lost to follow-up by letter or telephone.

Clinical and pathological data of patients were prospectively collected. The data include: (a) social demographics: age, marital status, education, and job; (b) tumor characteristics: tumor size, histological type, lymph nodes status, tumor staging, and location of the tumor; (c) treatment-related characteristics: number of axillary lymph nodes removed, type of surgery, surgical infection/ seroma/early edema, level of ALND, radiotherapy, chemotherapy and hormonal therapy; and (d) history of hypertension and body mass index.

LE was defined by circumferential measurement taken at 4 points: the metacarpal-phalangeal joints, the wrists, $10 \mathrm{~cm}$ below the lateral epicondyle and $15 \mathrm{~cm}$ above the lateral epicondyle [13]. The difference of $2 \mathrm{~cm}$ or more at any level compared with the opposite upper extremity was regarded as LE.

Categorical data were summarized as percentages; continuous variables were presented as mean with standard deviation (SD). Univariate comparisons were carried out using the Chi-square test for qualitative variables. Characteristics that were found to be significant by univariate analysis were then entered in a forward stepwise method in a logistical regression analysis to develop a multivariate model of independent factors for predicting the presence of LE.

A scoring system was developed with: (a) a ß-coefficient obtained for each significant factor from the multivariate logistical regression model; (b) each of the coefficient variables rounded off to a whole integer; and (c) the score of each variable set as its corresponding coefficient $[14,15]$. The cut-off values, sensitivity, and specificity of each score were determined using the receiver operating characteristic (ROC) curve method. Calibration was studied from graphical representations of the relationship between the observed outcome frequencies and the predicted probabilities (calibration curves). The internal validation used bootstrapping techniques. 200 bootstrap samples were drawn from the original data set with replacement [16].

Statistical analyses were performed using SPSS statistical software (version 10.0, SPSS Inc., Chicago, IL) and Excel (2003). All tests were two-sided with $\mathrm{p}$ values of 0.05 being considered as significant.

\section{Results}

Of the 378 patients were recruited at baseline, 20 dropped out of the study including 5 withdrawals after 5 weeks due to job changes, 2 died, and 12 did not return follow-up questionnaires. Thus, 358 Patients were analyzed. There was no statistically significant difference for any variables between the 20 lost patients and the remaining 358 patients. Of the analyzed cohort, 114 (31.84\%) developed LE. Descriptive characteristics of the 358 patients are shown in table 1 .

History of hypertension, axillary lymph node status, surgery on dominant arm, surgical infection/early edema/seroma, level of ALND, number of lymph nodes dissected, and radiotherapy were significant risk factors in univariate analysis $(\mathrm{p}<0.05)$ (table 1). All of the factors with a $\mathrm{p}$ value of less than 0.05 were included in the multivariate analysis. The results of multivariate stepwise logistic regression are shown in table 2 . History of hypertension, surgery 
Table 3. Points for predictive factors

\begin{tabular}{ll}
\hline Predictor variable & Points \\
\hline Hypertension & \\
$\quad$ No & 0 \\
$\quad$ Yes & 1 \\
Surgery on dominant arm & 0 \\
$\quad$ No & 1 \\
$\quad$ Yes & 0 \\
Level of ALND & 1 \\
I & 2 \\
II & \\
III & 0 \\
Radiotherapyl & 2 \\
$\quad$ No & \\
$\quad$ Yes (breast/chest wall or lymph & \\
node basins) & \\
Surgical infection/seroma/early edema & \\
$\quad$ No & 0 \\
$\quad$ Yes & 2 \\
\hline ALND = axillary lymph node dissection & \\
\hline
\end{tabular}

on dominant arm, surgical infection/early edema/seroma, level of ALND, and radiotherapy were independent risk factors.

Based on the multivariate analysis, a scoring system (table 3 ) was developed to predict individual probability of LE after ALND in breast cancer. Variables that were associated with LE and their corresponding score in scoring system were: the level of ALND (level I $=0$, level II $=1$, and level III $=2$ ), history of hypertension (yes $=1$, no $=0$ ), surgery on dominant arm (yes $=1$, no $=0$ ), radiotherapy (yes $=2$, no $=0$ ), and surgical infection/seroma/early edema (yes $=2$, no $=0$ ).

Figure 1 shows the ROC curve corresponding to the multivariate logistic model. The area under the ROC curve (AUC) was 0.877 , indicating the potentially promising predictive power of the multivariate logistic regression model. If a cut-off value of 3 was used, the sensitivity was $81.20 \%$ and the specificity was $80.90 \%$. The AUC, corrected by bootstrapping, was 0.798 .

The calibration of the internal validation was good as shown in figure 2. The $\mathrm{x}$-axis is the prediction calculated from the scoring system and the $y$-axis is the actual LE observed in the cohort. The reference line indicates the difference between the predicted probabilities. The calibration plots showed a minute departure from ideal predictions.

\section{Discussion}

Few of the different statistical models proposed in breast cancer have addressed LE. We used multivariate logistic regression analyses to eliminate confounding variables and to obtain a concise set of important and independent variables to be included in our scoring system. Our tool shares the same advantages as other predictive models, such as nomograms, based on multivariable statistical approaches, but with it, it is easier to generate a final score relating to LE risk. This method does not require a calculator or computer

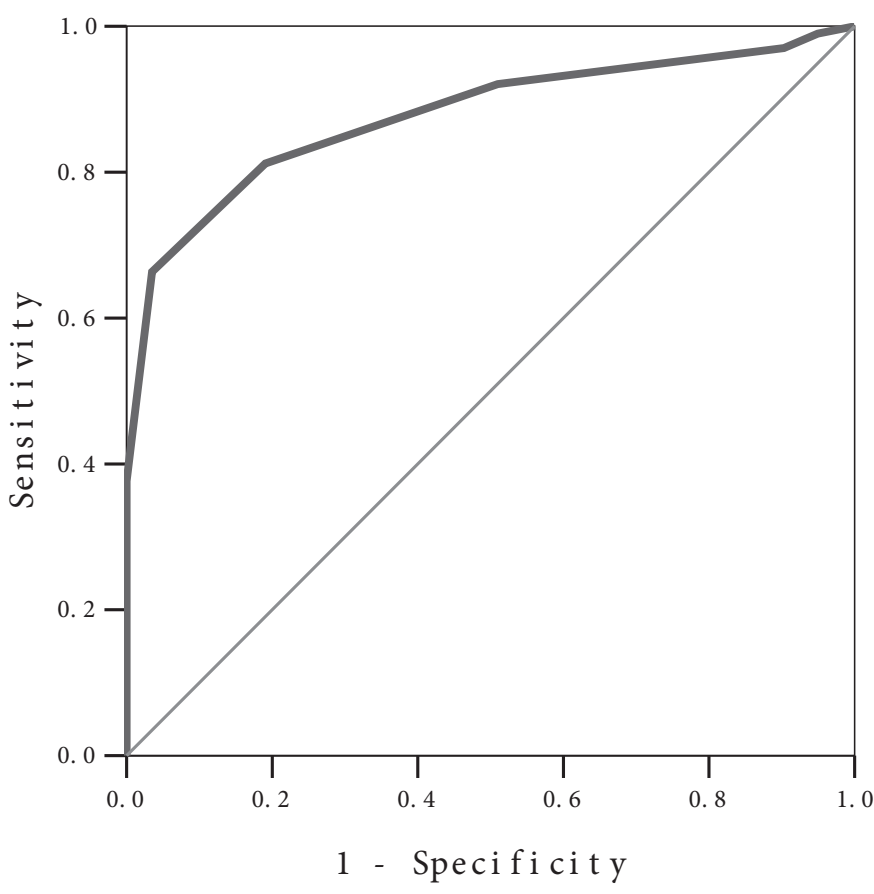

Fig. 1. Receiver operating characteristic curve corresponding to the multiple logistic models. Diagonal segments are produced by ties.

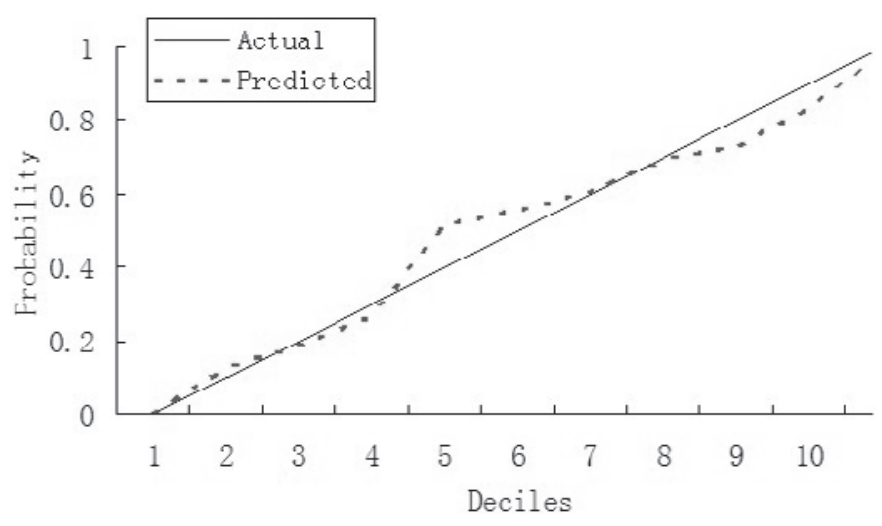

Fig. 2. Calibration plot comparing actual and predicted probabilities of lymphedema.

and simplifies the estimation of risk based on complex statistical models [14].

The scoring system that we have developed is composed of 5 clinic pathological factors: the level of ALND, radiotherapy, surgical infection/seroma/early edema, history of hypertension, and surgery on dominant arm. The 2 most heavily weighted factors were the level of ALND and radiotherapy to the lymph node basin or breast/chest wall. They were demonstrated as independent predictors of LE in a meta-analysis performed by Tsai et al. [17]. Few studies have reported an association between LE and hypertension. In our study, women with a history of hypertension were 7-fold more likely to develop arm LE than those with no such history (odds ratio (OR) 2.753; 95\% confidence interval (CI) 1.343-5.642). 
This may due to the fact that high capillary pressure facilitates leakage of fluid into the tissues.

Physical activities such as the level of exercise and LE-risk reduction behavior were not included in our study. If we integrate these predictor variables, our model may be more accurate. Surgery on dominant arm was risk factor for LE (OR 2.454 (1.0125.948)) in our study, but Clark [18] stated that surgery on the dominant arm was a protective factor for LE (OR 0.74 (0.41-1.31)). Our explanation of this phenomenon is that the patients in our study were not given sufficient education about LE prevention and excise, and thus they were not able to handle the intensity of excise. Our recommendation is to perform moderate strengthening and stretching exercises to maintaining muscle strength, but avoid too vigorous, repetitive, or excessive upper body exercises.

Some variables have long been recognized as risk factors for LE, e.g. tumor size, tumor stage, axillary lymph node status, type of surgery, and number of axillary lymph nodes removed [18, 19]. However, those factors were not found to be independent predictors in our study. Nor was arm LE associated with education or income level and marital status.

The discrimination of our scoring system in distinguishing patients with different outcomes was good, with an AUC of 0.877 (95\% CI, 0.830-0.924). This means that, given 2 randomly selected patients, 1 of whom has no LE and the other not, there is a $76 \%$ chance that the scoring system will predict a patient with a higher probability for developing subsequent LE. The cut-off value for scoring system is 3 .

As $89.9 \%$ of the patients in our cohort had undergone modified radical mastectomy with ALND, few patients had received breastconserving surgery and none of the patients had undergone SLN surgery. Thus, our scoring system might not be appropriate in other validation datasets. Further work is needed to validate this system in much larger, multi-center cohorts. Variations in pathological assessment between centers are one of the major impediments to widespread application of scoring systems. Despite the limitations mentioned above our scoring system could be a simple and easy tool for both the physician and patients to estimate the risk of LE.

\section{Acknowledgments}

This work was supported by grants from the National Natural Science Foundation of China (81172201)

\section{Disclosure Statement}

The authors declare that they have no conflict of interest.

\section{References}

1 Nielsen I, Gordon S, Selby A: Breast cancer-related lymphoedema risk reduction advice: A challenge for health professionals. Cancer Treat Rev 2008;34:621628.

2 Jemal A, Siegel R, Ward E, et al.: Cancer statistics, 2008. CA Cancer J Clin 2008;58:71-96.

3 Giuliano AE, McCall L, Beitsch P, et al.: Locoregional recurrence after sentinel lymph node dissection with or without axillary dissection in patients with sentinel lymph node metastases: The American College of Surgeons Oncology Group Z0011 randomized trial. Ann Surg 2010;252:426-432.

4 Rietman JS, Dijkstra PU, Geertzen JH, et al.: Shortterm morbidity of the upper limb after sentinel lymph node biopsy or axillary lymph node dissection for Stage I or II breast carcinoma. Cancer 2003;98:690696.

5 Mansel RE, Fallowfield L, Kissin M, et al.: Randomized multicenter trial of sentinel node biopsy versus standard axillary treatment in operable breast cancer: The ALMANAC Trial. J Natl Cancer Inst 2006;98:599-609.

6 Fu MR, Ridner SH, Armer J: Post-breast cancer. Lymphedema: part 1. Am J Nurs 2009;109:48-54; quiz 55.
7 Dahl AA, Nesvold IL, Reinertsen KV, et al.: Arm/ shoulder problems and insomnia symptoms in breast cancer survivors: Cross-sectional, controlled and longitudinal observations. Sleep Med 2011;12:584-590.

8 Rock CL, Demark-Wahnefried W: Can lifestyle modification increase survival in women diagnosed with breast cancer? J Nutr 2002;132:3504S-3507S.

9 Hinrichs CS, Watroba NL, Rezaishiraz H, et al.: Lymphedema secondary to postmastectomy radiation: Incidence and risk factors. Ann Surg Oncol 2004;11: 573-580.

10 Armer JM, Stewart BR: Post-breast cancer lymphedema: Incidence increases from 12 to 30 to 60 months. Lymphology 2010;43:118-127.

11 Sakorafas GH, Peros G, Cataliotti L, et al.: Lymphedema following axillary lymph node dissection for breast cancer. Surg Oncol 2006;15:153-165.

12 Vignes S, Porcher R, Arrault M, et al.: Long-term management of breast cancer-related lymphedema after intensive decongestive physiotherapy. Breast Cancer Res Treat 2007;10:285-290.

13 Harris SR, Hugi MR, Olivotto IA, et al.: Clinical practice guidelines for the care and treatment of breast cancer: 11. Lymphedema. CMAJ 2001;164:191-199.
14 Sullivan LM, Massaro JM, D’Agostino RB Sr: Presentation of multivariate data for clinical use: The Framingham Study risk score functions. Stat Med 2004;23: 1631-1660.

15 Indarti J, Aziz MF, Suryawati B, et al.: Scoring system and management algorithm assessing the role of survivin expression in predicting progressivity of HPV infections in precancerous cervical lesions. Asian Pac J Cancer Prev 2013;14:1643-1647.

16 Steyerberg EW: Clinical prediction models: A practical approach to development, validation, and updating. New York, NY: Springer, 2009.

17 Tsai RJ, Dennis LK, Lynch CF, et al.: The risk of developing arm lymphedema among breast cancer survivors: A meta-analysis of treatment factors. Ann Surg Oncol 2009;16:1959-1972.

18 Clark B, Sitzia J, Harlow W: Incidence and risk of arm oedema following treatment for breast cancer: a threeyear follow-up study. QJM 2005;98:343-348.

19 Park JH, Lee WH, Chung HS: Incidence and risk factors of breast cancer lymphoedema. J Clin Nurs 2007; 17:1450-1459. 